\title{
Color-converting combinations of nanocrystal emitters for warm-white light generation with high color rendering index
}

\author{
Sedat Nizamoglu, Gulis Zengin, and Hilmi Volkan Demir ${ }^{a)}$ \\ Department of Electrical and Electronics Engineering, Department of Physics, and Nanotechnology \\ Research Center, Bilkent University, Ankara TR-06800, Turkey
}

(Received 29 November 2007; accepted 19 December 2007; published online 22 January 2008)

\begin{abstract}
Warm-white light emitting diodes with high color rendering indices are required for the widespread use of solid state lighting especially indoors. To meet these requirements, we propose and demonstrate warm-white hybrid light sources that incorporate the right color-converting combinations of $\mathrm{CdSe} / \mathrm{ZnS}$ core-shell nanocrystals hybridized on InGaN/GaN LEDs for high color rendering index. Three sets of proof-of-concept devices are developed to generate high-quality warm-white light with (1) tristimulus coordinates $(x, y)=(0.37,0.30)$, luminous efficacy (LE) $=307 \mathrm{~lm} / \mathrm{W}$, color rending index $(\mathrm{CR})=82.4$, and correlated color temperature $(\mathrm{CCT})=3228 \mathrm{~K} ;(2)$ $(x, y)=(0.38,0.31), \mathrm{LE}=323 \mathrm{~lm} / \mathrm{W}, \mathrm{CRI}=81.0$, and $\mathrm{CCT}=3190 \mathrm{~K}$; and $(3)(x, y)=(0.37,0.30)$, $\mathrm{LE}=303 \mathrm{~lm} / \mathrm{W}, \mathrm{CRI}=79.6$, and CCT=1982 K. (C) 2008 American Institute of Physics.

[DOI: $10.1063 / 1.2833693$ ]
\end{abstract}

Climate change is considered to be one of the major issues that humankind faces in our century. Today, the emission rate of greenhouse gases including carbon dioxide is on an alarmingly rapid rise around the globe. ${ }^{1}$ The widespread use of solid state based lighting (SSL) is of great importance to significantly reduce the global electricity consumption and the use of fossil fuels. ${ }^{2}$ Today, $20 \%$ of the electricity is globally consumed for lighting; for indoor applications (homes, offices, etc.), the lighting is responsible for up to $50 \%$ of the total energy consumption. ${ }^{2,3} \mathrm{SSL}$ is predicted to save $50 \%$ of the electricity consumption for lighting and reduce the carbon emission by $300 \times 10^{6}$ tons annually. ${ }^{2,4}$ Therefore, SSL offers an effective way to deal with the challenge of reducing greenhouse gas emission and combating climate change. Today, the most commonly used SSL sources are based on the integration of yttrium aluminum garnet (YAG) phosphors on blue InGaN/GaN light emitting diodes (LEDs). ${ }^{5,6}$ The broad yellowish emission of YAG phosphors along with blue LED yields white light generation with correlated color temperatures (CCTs) of 4000-8000 K, corresponding to the neutraland cool-white intervals, and color rendering indices (CRIs) typically lower than $80 .^{7,8}$ However, especially for widescale use in indoor illumination applications, white LEDs (WLEDs) are required to provide warm enough CCT $(<4000 \mathrm{~K})$ with high enough CRI $(>80))^{4,7,8}$

Recently, nanocrystal (NC) based optoelectronic devices have made great progress in device research. ${ }^{9-21}$ Nanocrystal emitters are particularly advantageous for use in white light sources because they feature tunable and relatively narrow emission across the visible spectral range and small overlap between their emission and absorption spectra, and also provide the ability to be easily and uniformly deposited in solid films with common techniques (e.g., spin casting and dip coating). In the previous reports, white light generation using $\mathrm{CdSe} / \mathrm{ZnS}$ core-shell nanocrystals of single, dual, trio, and quadruple combinations on blue InGaN/GaN LEDs have been demonstrated. ${ }^{15} \mathrm{~A}$ blue/green two-wavelength InGaN/GaN LED coated with a single type of red NC and

${ }^{a)}$ Electronic mail: volkan@bilkent.edu.tr. Tel.: (+90)(312) 290-1021. FAX: $(+90)(312)$ 290-1015. a blue InGaN/GaN LED with a single type of yellow NC and a dual type of red and green NCs have been also reported. $^{16,17}$ In our previous work, white light generation with high color rendering index $>80$ using dual hybridization of nanocrystals and polymers on LEDs has been achieved. ${ }^{18}$ Additionally, WLEDs have been realized by integrating NCs with polymethylmethacrylate (PMMA) on ultraviolet LEDs. ${ }^{19-21}$ These NC-based white LEDs have been shown to exhibit high CRI. However, in the previous studies of our group and others, using such high-CRI nanocrystalbased hybrid LEDs, warm correlated color temperature along with high CRI has not been demonstrated to date, although such high-quality white light is required in the future according to the SSL roadmap. $4,7,8$

In this letter, we present nanocrystal-based warm-white hybrid light sources with high color rendering index that incorporate the right color-converting combinations of green and red $\mathrm{CdSe} / \mathrm{ZnS}$ core-shell nanocrystals (emitting at $\lambda_{\mathrm{PL}}$ $=555$ and $613 \mathrm{~nm}$, respectively) hybridized on blue InGaN/GaN light emitting diodes (at $\lambda_{\mathrm{EL}}=452 \mathrm{~nm}$ ). The use of such nanocrystal emitters facilitates achieving high correlated color temperature while maintaining the chromaticity operating point within the white region and keeping color rendering index high. This is primarily because nanocrystals have relatively narrow emission in the visible (e.g., full width at half maximum $<30 \mathrm{~nm}$ in solution) and their peak emission wavelength can be fine tuned with the size effect as necessary. Therefore, using a right color-converting combination of nanocrystals, it is possible in principle to generate and adjust any emission spectrum as desired. Also, in the case of using such nanocrystal emitters, the red emission above $650 \mathrm{~nm}$ can be significantly avoided, unlike using the phosphors, which exhibits strong emission tail in the red above $650 \mathrm{~nm}$ and reduces its luminous efficacy (LE) (because of the eye sensitivity function decreasing quickly above $650 \mathrm{~nm}$ ). The hybrid NC-LED luminescence can thus be carefully tuned by taking into account the eye sensitivity function to achieve high luminous efficacy. Based on our careful designs and hybridization of the nanocrystal emitters, we develop and demonstrate three sets of proof-of-concept warm-white LEDs with high-quality white light properties: 


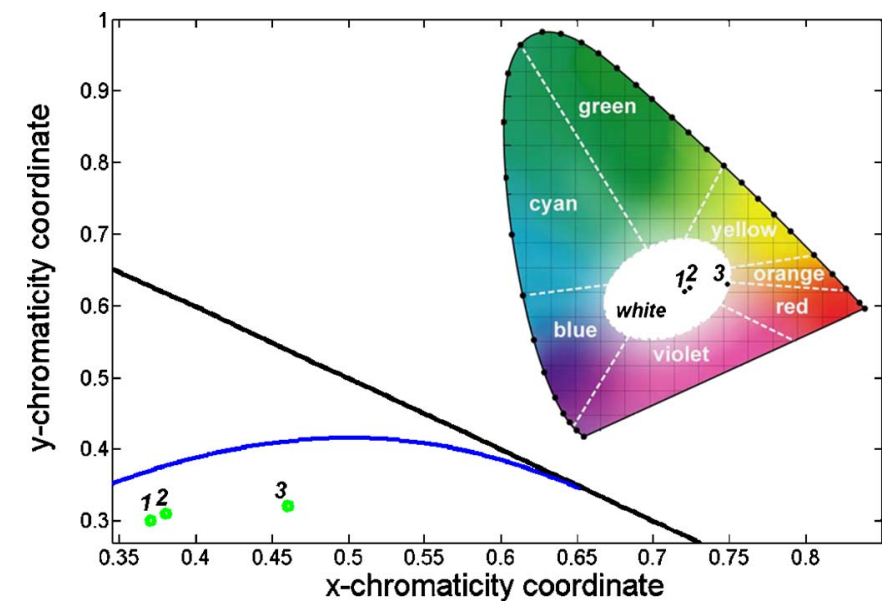

FIG. 1. (Color online) CIE chromaticity diagram zoomed-in for the loci of the tristimulus coordinates of our nanocrystal-hybridized warm-white light emitting diodes (green points) along with the planckian locus (blue line). A complete CIE 1931 chromaticity diagram, e.g., as in Ref. 5, is also given with the tristimulus coordinates of our hybrid warm-white light emitting diodes in the inset.

(1) the tristimulus coordinates $(x, y)=(0.37,0.30)$, LE $=307 \mathrm{~lm} / \mathrm{W}, \mathrm{CRI}=82.4$, and $\mathrm{CCT}=3228 \mathrm{~K} ;(2)(x, y)$ $=(0.38,0.31), \quad \mathrm{LE}=323 \mathrm{~lm} / \mathrm{W}, \quad \mathrm{CRI}=81.0, \quad$ and $\mathrm{CCT}$ $=3190 \mathrm{~K}$; and $(3)(x, y)=(0.37,0.30), \mathrm{LE}=303 \mathrm{~lm} / \mathrm{W}$, CRI $=79.6$, and CCT $=1982 \mathrm{~K}$, as shown on the CIE 1931 chromaticity diagram in Fig. 1.

The operating principle of these hybrid NC-WLEDs is based on the mutual use of the integrated NC film as the photoluminescent layer and the LED as the pump light source. The integrating LED platform optically excites the $\mathrm{NC}$ emitters when it is electrically driven. Consequently, the $\mathrm{NC}$ photoluminescence and the LED electroluminescence collectively contribute to the white light generation. We use InGaN/GaN based blue light emitting platform as the excitation source at $452 \mathrm{~nm}$. We design and grow the epitaxial structure of these InGaN/GaN LEDs and fabricate them using the standard microfabrication techniques similar to those described in our previous work. ${ }^{15,22-25}$ Such InGaN/GaN LEDs are demonstrated to achieve long lifetime (ten thousands of hours). ${ }^{8}$ To make the hybrid warm-WLEDs, we integrate green- and red-emitting $\mathrm{CdSe} / \mathrm{ZnS}$ core-shell NCs (at $\lambda_{\mathrm{PL}}=555$ and $613 \mathrm{~nm}$, respectively) in the PMMA matrix on top of the blue LEDs. The optical properties of these nanocrystals and their hybridization method are also explained in our previous work. ${ }^{17}$ Such nanocrystal emitters are investigated to study their photostability; typical shelf lifetime of these nanocrystals is reported to be thousands of hours. ${ }^{26}$ To obtain white light generation with warm color temperature and high color rendering index, we analyze the blackbody radiators on the planckian locus of CIE chromaticity diagram, which are used as the reference sources. Based on this analysis, we set the correct amount of NC emitters for the LED hybridization to achieve high performance. In Fig. 2, we show the spectrum of blackbody radiators at the color temperature of the fabricated hybrid warm-white light emitting diodes (samples 1-3). As the color temperature of the radiators decreases (getting warmer in color), the red part in the visible becomes more dominant. Therefore, to achieve warmer color temperatures, we increase the red luminescence in the visible spectrum, while maintaining the chromaticity point in the white region and sustaining high color

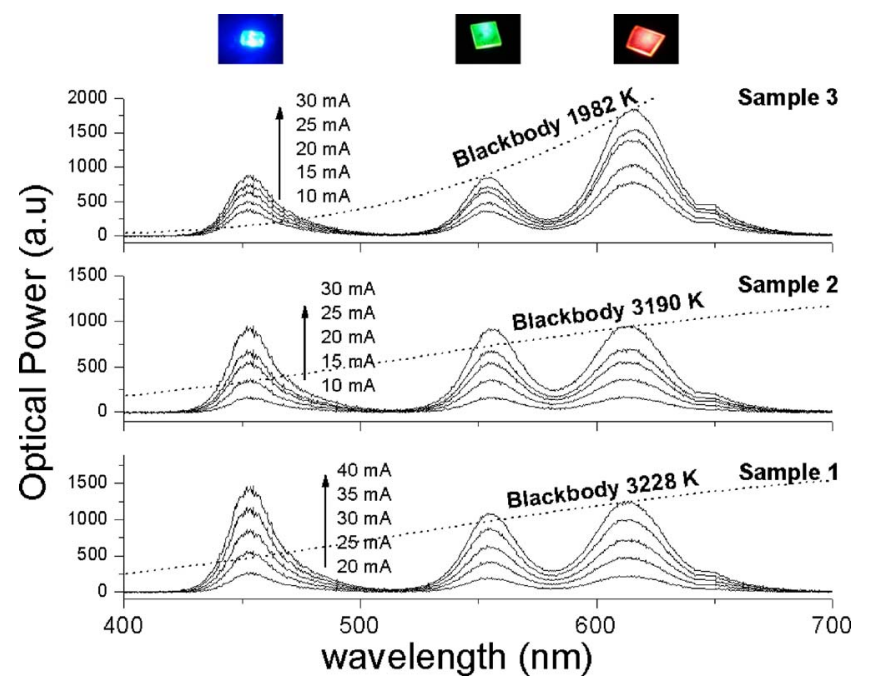

FIG. 2. (Color online) Luminescence spectra of our nanocrystal hybridized warm-white light emitting diodes (samples 1-3).

rendering index. For the optical characterization of the resulting integrated NC WLEDs, we obtain the operating chromaticity coordinates, the correlated color temperature, the color rendering index, and the optical luminous efficacy, as explained in detail in Ref. 5.

In the first experimental proof-of-concept demonstration, for their hybridization on blue LED $\left(\lambda_{\mathrm{EL}}=452 \mathrm{~nm}\right)$, we design to incorporate $0.22 \mathrm{mg}(0.578 \mathrm{nmole})$ of red-emitting $\mathrm{CdSe} / \mathrm{ZnS}$ core-shell NCs and, subsequently, $0.26 \mathrm{mg}$ (2.166 nmole) of green-emitting NCs. These nanocrystals are selected with 9.6 and $7.7 \mathrm{~nm}$ diameters (with a size distribution of $\pm 5 \%$ ) to emit at the peak wavelengths of 613 and $555 \mathrm{~nm}$, respectively. These red nanocrystal emitters are carefully chosen to provide sufficiently red emission to increase the color temperature, which is not too red in color to contribute significantly to emission above $650 \mathrm{~nm}$ and undesirably reduce the luminous efficacy. On the other hand, the green nanocrystal emitters are chosen to balance out the red emission conveniently at $555 \mathrm{~nm}$ along with the blue LED emission at $452 \mathrm{~nm}$ and, consequently, keep the operating chromaticity coordinates within the white region and the color rendering index high enough. We obtain the luminescence of the resulting hybrid LED at various current injection levels, as shown in Fig. 2. As the injected current increases, the luminescence of the hybrid LED increases, while maintaining the relative peak levels in blue, green, and red. At all current injection levels, the emission of the LED leads to $(x, y)=(0.37,0.30), \quad \mathrm{LE}=307 \mathrm{~lm} / \mathrm{W}, \quad \mathrm{CRI}=82.4$, and $\mathrm{CCT}=3228 \mathrm{~K}$. This corresponds to a warm-white LED with a high color rendering index of 82.4, satisfying the future SSL criterion of $\mathrm{CRI} \sim 80$. $^{4,7,8}$

For the second demonstration, we design to integrate $0.13 \mathrm{mg}$ (1.083 nmole) of green-emitting $\mathrm{CdSe} / \mathrm{ZnS}$ coreshell NCs $\left(\lambda_{\mathrm{PL}}=555 \mathrm{~nm}\right)$ and then $0.44 \mathrm{mg}(1.156 \mathrm{nmole})$ of red-emitting $\mathrm{NCs}\left(\lambda_{\mathrm{PL}}=613 \mathrm{~nm}\right)$ on the top of blue LED $\left(\lambda_{\mathrm{EL}}=452 \mathrm{~nm}\right)$. Again, the nanocrystal emitters are carefully chosen to mimic the optical spectrum of the associated blackbody radiator as much as possible. We show the luminescence of the hybrid LED at various current injection levels in Fig. 2. This implementation experimentally leads to $(x, y)=(0.38,0.31), \mathrm{LE}=323 \mathrm{~lm} / \mathrm{W}, \mathrm{CRI}=81.0$, and CCT $=3190 \mathrm{~K}$. Here, the tristimulus coordinates shift to the red 
TABLE I. Optical properties of our nanocrystal hybridized warm-white light emitting diodes.

\begin{tabular}{cccccc}
\hline \hline Sample & $x$ & $y$ & LE $(\mathrm{lm} / \mathrm{W})$ & CRI & CCT (K) \\
\hline 1 & 0.37 & 0.30 & 307 & 82.4 & 3228 \\
2 & 0.38 & 0.31 & 323 & 81.0 & 3190 \\
3 & 0.46 & 0.32 & 303 & 79.6 & 1982 \\
\hline \hline
\end{tabular}

side of the CIE chromaticity diagram and the correlated color temperature decreases to $3190 \mathrm{~K}$ because of the increased relative intensity of the red-emitting nanocrystals. Therefore, this light source achieves a warmer-white light generation while maintaining its operation in white. The color rendering index slightly drops to 81.0 , which still satisfies the criterion for the future SSL sources, and the luminous efficacy reaches a relatively high value of $323 \mathrm{~lm} / \mathrm{W}$.

As the last demonstration, we design to hybridize $0.13 \mathrm{mg}$ (1.083 nmole) of green-emitting $\mathrm{CdSe} / \mathrm{ZnS}$ coreshell NCs $\left(\lambda_{\mathrm{PL}}=555 \mathrm{~nm}\right)$ and $0.66 \mathrm{mg}(1.734 \mathrm{nmole})$ of redemitting $\mathrm{NCs} \quad\left(\lambda_{\mathrm{PL}}=613 \mathrm{~nm}\right)$ on the blue LED $\left(\lambda_{\mathrm{EL}}\right.$ $=452 \mathrm{~nm}$ ). The resulting emission spectra at various levels of current injection are shown in Fig. 2, corresponding to $(x, y)=(0.37,0.30), \mathrm{LE}=303 \mathrm{~lm} / \mathrm{W}, \mathrm{CRI}=79.6$, and $\mathrm{CCT}$ $=1982 \mathrm{~K}$. This operating point stands approximately on the boundary of white region near to the red-color end, as shown in Fig. 1. Therefore, this hybrid white LED generates highly warm-white light at an extralow correlated color temperature of $1982 \mathrm{~K}$.

Hybridizing $\mathrm{CdSe} / \mathrm{ZnS}$ core-shell $\mathrm{NC}$ emitters on InGaN/GaN based blue LEDs, we demonstrate three warmwhite light sources with CCT ranging from 3227 to $1982 \mathrm{~K}$. In these proof-of-concept demonstrations, the color rendering indices as high as 82.4 and luminous efficacies as high as $327 \mathrm{~lm} / \mathrm{W}$ are achieved. Table I provides a list of these hybrid nanocrystal-based warm-white light emitting diodes along with their corresponding $(x, y)$ coordinates, LE, CRI, and CCT.

In conclusion, we presented nanocrystal-hybridized warm-white light emitting diodes with high color rendering index and high luminous efficacy. In this work, the use of nanocrystal emitters in the right color-converting combinations enabled such hybrid white light sources to achieve highly warm correlated color temperature, while maintaining their operating chromaticity coordinates in the white region and sustaining their high color rendering index. Our proofof-concept demonstrations indicate that such nanocrystalbased warm-white light emitting diodes with high-quality white light properties hold great promise especially for future indoor lighting applications.

This work is supported by EU-PHOREMOST No. E511616 and Marie Curie European Reintegration Grant MOON 021391 and TUBITAK under the Project Nos. EEEAG 106E020, 104E114, 107E080, 105E065, and 105E066.
Also, H.V.D. acknowledges additional support from the Turkish Academy of Sciences Distinguished Young Scientist Award (TUBA GEBIP) and European Science Foundation (ESF) European Young Investigator Award (EURYI) Programs. The authors are also pleased to acknowledge the use of the facilities of Bilkent University Nanotechnology Research Center (founder Professor E. Ozbay) and Advanced Research Laboratories (founder Professor S. Ciraci).

${ }^{1}$ Intergovernmental Panel on Climate Change (IPCC) Human and Natural Drivers of Climate Change 2007 Report, Summary for Policymakers, 2007 (unpublished), pp. 1-18.

${ }^{2}$ The Promise of Solid State Lighting for General Illumination Light Emitting Diodes (LEDs) and Organic Light Emitting Diodes (OLEDs), Optoelectronics Industry Development Association, Washington, DC, see (http://www.netl.doe.gov/ssl/PDFs/oida_led-oled_rpt.pdf).

${ }^{3}$ U.S. Environmental Protection Agency (EPA) Greenhouse Gas Inventory 1990-2005 Reports, Carbon Dioxide Emissions, Executive Summary, 2007 (unpublished), pp. 6-7.

${ }^{4}$ Solid State Lighting, Sandia National Laboratories, see (http:// lighting.sandia.gov).

${ }^{5}$ E. F. Schubert, Light Emitting Diodes (Cambridge University Press, New York, 2006).

${ }^{6}$ S. Nakamura and G. Fasol, The Blue Laser Diode (Springer, Berlin, 1997), pp. 216-219.

${ }^{7}$ M. R. Krames, O. B. Shchekin, R. Mueller-Mach, G. O. Mueller, L. Zhou, G. Harbers, and M. G. Craford, J. Disp. Technol. 3, 2 (2007).

${ }^{8}$ J. Y. Tsao, IEEE Circuits Devices Mag. 20, 3 (2004).

${ }^{9}$ N. P. Gaponik, D. V. Talapin, and A. L. Rogach, Phys. Chem. Chem. Phys. 1, 1787 (1999).

${ }^{10}$ S. Sapra, S. Mayilo, T. A. Klar, A. L. Rogach, and J. Feldmann, Adv. Mater. (Weinheim, Ger.) 19, 569 (2007).

${ }^{11}$ A. M. R. Hikmet, T. K. P. Chin, D. V. Talapin, and H. Weller, Adv. Mater. (Weinheim, Ger.) 17, 1436 (2005).

${ }^{12}$ J. H. Ahn, C. Bertoni, S. Dunn, C. Wang, D. V. Talapin, N. Gaponik, A. Eychmüller, Y. Hua, M. R. Bryce, and M. C. Petty, Nanotechnology 18, 335202 (2007).

${ }^{13}$ E. Mutlugun, I. M. Soganci, and H. V. Demir, Opt. Express 15, 1128 (2007).

${ }^{14}$ I. M. Soganci, S. Nizamoglu, E. Mutlugun, O. Akin, and H. V. Demir, Opt. Express 15, 14289 (2007).

${ }^{15}$ S. Nizamoglu, T. Ozel, E. Sari, and H. V. Demir, Nanotechnology 18, 065709 (2007)

${ }^{16}$ H. Chen, D. Yeh, C. Lu, C. Huang, W. Shiao, J. Huang, C. C. Yang, I. Liu, and W. Su, IEEE Photonics Technol. Lett. 18, 1430 (2006).

${ }^{17}$ H. Chen, C. Hsu, and H. Hong, IEEE Photonics Technol. Lett. 18, 193 (2006).

${ }^{18}$ H. V. Demir, S. Nizamoglu, T. Ozel, E. Mutlugun, I. O. Huyal, E. Sari, E. Holder, and N. Tian, New J. Phys. 9, 362 (2007).

${ }^{19}$ M. Ali, S. Chattopadhyay, A. Nag, A. Kumar, S. Sapra, S. Chakraborty, and D. D. Sarma, Nanotechnology 18, 075401 (2007).

${ }^{20}$ S. Nizamoglu and H. V. Demir, J. Opt. A, Pure Appl. Opt. 9, S419 (2007).

${ }^{21}$ S. Nizamoglu and H. V. Demir, Nanotechnology 18, 405702 (2007).

${ }^{22}$ E. Sari, S. Nizamoglu, T. Ozel, and H. V. Demir, Appl. Phys. Lett. 90, 011101 (2007).

${ }^{23}$ V. A. Sabnis, H. V. Demir, O. Fidaner, J. S. Harris, D. A. B. Miller, J. F. Zheng, N. Li, T. C. Wu, H. T. Chen, and Y. M. Houng, Appl. Phys. Lett. 84, 469 (2004).

${ }^{24}$ H. V. Demir, V. A. Sabnis, J. F. Zheng, O. Fidaner, J. S. Harris, and D. A. B. Miller, IEEE Photonics Technol. Lett. 16, 2305 (2004).

${ }^{25}$ T. Ozel, E. Sari, S. Nizamoglu, and H. V. Demir, J. Appl. Phys. 102, 113101 (2007)

${ }^{26}$ Evident Technologies, see (http://www.evidenttech.com/products/evidots/ evidot-specifications.html? searched $=$ shelf + lifetime $\&=$ ajaxSearch _highlight + ajaxSearch_highlight1 + ajaxSearch_highlight2), 2007. 